\title{
Does Mass Shooting Influence Attitudinal Change? New Evidence from Orlando 2016
}

\author{
Muzhou Zhang ${ }^{1, *}$ and Joseph Kelly ${ }^{1}$ \\ ${ }^{1}$ Department of Government, University of Essex, UK \\ ${ }^{*}$ Corresponding author. Email: muzhou.zhang@essex.ac.uk
}

October 12,2020

\begin{abstract}
Does mass shooting event influence gun control attitudes? Previous research conducted by Rogowski and Tucker (2019) suggests that attitudes towards gun control remained unchanged following the 2012 Sandy Hook Elementary School shooting. Given this finding's counterintuitive nature and significance, we further investigate gun control attitudinal change using an exogenous event. Here, we employ a similar method while using the 2016 Orlando shooting as a new case to explore the extent to which the previously reported null effect holds. By leveraging the pre- and post-shooting responses for wave 55 of The American Panel Survey, we show that American's gun control attitudes are unmalleable and deeply entrenched following mass shooting events. Our null finding has meaningful implications for the deadlocked gun control issue in the US.
\end{abstract}

1. This work is based on an assignment of GV915-7-SU Applied Research Design (2010-2020) at the University of Essex. We are grateful to Carolina Garriga and Seonghui Lee for helpful suggestions. All errors are our own. The replication files are available at https://github . com/muzhouzhang/orlando. 


\section{Introduction}

Bloody mass shootings repeatedly happen in the US while shocking the world. Compared to Australia, Canada, Great Britain, and New Zealand, which all experienced horrific gun-related massacres and then introduced legislation to tighten gun control, ${ }^{1}$ there is no significant gun control progress in the US. In representative democracies, public support is, though not necessarily, an important factor to push policy changes. The deadlocked gun control issue in the US leads us to ask are mass shootings able to galvanize public support for gun control? Existing research on this very topic has produced contradictory results. Newman and Hartman (2019) argued that geographical proximity has an influential effect on support for increasing gun control following a mass shooting event. They found those who live within a 100-mile radius of a shooting event are more likely to prefer stricter laws covering the sale of firearms. In contrast, Barney and Schaffner (2019) later found this effect is null, even when varying the proximity threshold. In their response, Hartman and Newman (2019) contested the null finding, arguing that pre-treatment exposure to mass shootings matter and the geographical proximity to a mass shooting event does increase support for firearm restrictions. Using the 2012 Sandy Hook Elementary School shooting as a case, Rogowski and Tucker (2019, RT hereafter) found such a painful event, which took 26 people's lives (20 of them were children), failed to increase American's support for gun control. This striking null finding holds even for subgroups whose predispositions are more in favor of gun legislation, such as parents and Democrats.

The null effect reported by RT is counterintuitive while possessing both scholarly and practical importance. In this research note, we extend RT's work by using the 2016 Orlando nightclub shooting as a new but comparable case. Employing the same data source (The American Panel Study, TAPS) and a similar method-unexpected event during survey design (Muñoz, Falcó-Gimeno, and Hernández 2020), we corroborate RT's null finding. By using TAPS wave 55 data and comparing the respondents who completed the survey before (Pre-shooting) and after (Post-shooting) June 12, 2016, when the Orlando shooting happened, we find American people were not responsive enough to this tragic gun violence incident which left 49 dead and 53 wounded, no matter whether we use a bivariate specification or include control variables aiming for covariate imbal-

1. See Port Arthur 1996 (Australia), Nova Scotia 2020 (Canada), Dunblane 1996 (Great Britain), and Christchurch 2019 (New Zealand) 
ance between the two groups. Our null finding holds when we apply various criteria to narrow down our sample. We find the difference of gun control legislation support is not statistically significant between Pre-shooting and Post-shooting when we only include the respondents who (a) completed the survey only one day before and after June 12, (b) lived near Orlando (whose residency is in Florida, Georgia, and Alabama), (c) finished the questionnaire within a reasonable duration (15-60 minutes), and those who satisfy (a) $\cap(\mathrm{c})$ and $(\mathrm{b}) \cap(\mathrm{c})$.

\section{Research Design}

We employ the unexpected-event-during-survey design, which leverages mass shooting's exogenous timing and survey's staggered participation, to evaluate the effect of mass shooting on people's gun control support. By comparing the respondents who answered the survey questionnaire before and after the shooting by randomness, we are able to minimize the confounding factors that select certain respondents to be more exposed to shooting event. We select the 2016 Orlando shooting for three reasons. First, by its occurrence, it was the deadliest gun violence event in US history. Its severity and impact makes it comparable to the 2012 Sandy Hook shooting, the one used in RT. Second, the Orlando shooting happened in a presidential election year like the Sandy Hook shooting did so that our case selection largely guarantees that any result discrepancy between ours and RT's should not be driven by the difference of macro political context, such as gun control debate intensity. Third, the Orlando shooting occurred in mid-month. This enables Pre-shooting and Post-shooting to have a relatively equal sample size. ${ }^{2}$

Our data come from TAPS wave 55, which was conducted online mainly in June 2016-the month when the Orlando shooting happened. To measure American's support for gun control, our outcome variable, we uses a question asking: "Do you generally support or oppose gun control legislation?" The substantive answers to this question are binary: "Support" or "Oppose". We code "Support" as 1 in our analysis. Our treatment indicator is determined by whether a respondent finished the questionnaire before or after the day when the Orlando shooting happened. Those who answered the questionnaire on and after June 13 are in the Post-shooting (treatment) group, in which the treatment is the exposure to the real-happening tragic mass shooting event. Those

2. This is why we do not select the 2017 Las Vegas shooting which happened on October 1. 
who participated the survey on and before June 11 are in the pre-shooting (control) group. After importing the original survey data, we have 1704 observations in total. Filtering out the cases whose completion date or survey weight are missing and the respondents who participated the survey exactly on June 12 gives us 1519 observations remaining. Among them, 57.07\% are in the Pre-shooting group (Appendix Table 1).

The respondents in our sample are "as-if" randomly assigned into the Pre- or Post-shooting group so ideally, there would be a covariate balance between these two. We choose 6 variables, namely Female, Parent, Political interest, News everyday, Ideology, and Political knowledge, to check if our treatment and control groups are comparable in terms of these personal characteristics that are likely to affect one's self-exposure to gun violence incident and attitude towards gun control simultaneously. Political interest is ordered at four categories: "Not at all", "Slightly", "Somewhat", and "Very". (We drop "Refused".) News everyday refers to whether a respondent consumes news on a daily basis. Ideology is on a 6-point scale in which 1 means liberal while 6 means conservative. Among a battery of political knowledge questions in the questionnaire, we select the one asking the term limit of the US senators. We code those who correctly gave an answer of six-year as 1 while others, including "Don't know" and "Refused", as $0 .^{3}$ By conducting difference-in-means tests, we find in the Post-shooting group, there are more females, parents, people with more interest in politics, everyday news consumers, conservatives, and people with more political knowledge (Appendix Table 3). In this situation, ignoring these control variables would confound our results if any. Hence, besides our bivariate specification, which simply regresses our outcome variable on the treatment indicator, we also include a full specification that incorporate these controls.

In $\mathrm{RT}^{\prime}$ s article, they did a panel (first-difference) analysis that compared the same respondent's answer regarding gun control question between the two survey waves-one before and one after the shooting. Two considerations prevent us from using this approach along with our cross-sectional design (comparing the respondents within a same wave). First, after wave 55 (June 2016), our gun control question was then asked in wave 57 (August 2016). This one-month gap likely to diminish the treatment effect that some respondents received. Second, if we used the first-difference approach, we would compare the wave 55 respondents who answered the ques-

3. For this insensitive and straightforward political knowledge question, we argue there is no reason for a person who firmly knew the correct answer to give a "Don't know" or "Refused" response. 
tionnaire before June 12 and themselves in wave 57. As we've shown, however, those who completed the survey before the shooting date are not comparable to those who finished after the day in terms of their personal characteristics that are correlated to gun control support. This means we would suffer from non-random sample selection problem if we used the first-difference approach.

In our sample, $7 \%$ of the respondents chose "No Opinion" when asked for their gun control legislation support. If the exposure to the Orlando shooting led some respondents more or less likely to have an opinion regarding our gun control legislation support question, discarding these observations would bias our results. To alleviate this concern, we regress an binary outcome indicating whether a respondent has an opinion ("Support" or "Oppose" = 1, "No opinion" = 0) to our treatment indicator to see if the rate of having an opinion is different between the two groups. The logit model results show the treatment indicator is statistically insignificant, suggesting there is no statistically distinguishable difference in terms of the rate of "No opinion" answer among Pre-shooting and Post-shooting (Appendix Table 4). Therefore, we safely drop the "No opinion" observations from our sample. Only 1\% of the respondents refused to answer our gun control question and we simply filter them out the sample. After doing these two steps, we have a sample size of 1400 .

In an unexpected-event-during-survey design, whether respondents really absorb treatment is a concern. In our research context, the previously reported null finding might be driven by that the Post-shooting group respondents forgot the shock of tragic shooting when they answered the relevant question or they even did not pay enough attention to the event when it occurred. To address these two possibilities, we narrow our sample down temporally and spatially. For our Narrow time window sample, we only keep the respondents who answered the questionnaire one day before and after June 12 to make the Orlando shooting's treatment effect (if any) remains as much as possible for the treated. For our Proximity sample, we only keep the respondents who lived in Florida (where Orlando locates) and its two contiguous states: Georgia and Alabama. The rationale behind this restriction is local people might be more responsive to local incidents, as argued in Newman and Hartman (2019). For an online survey like TAPS, respondents' attentiveness is another common problem. In our sample, the minimum survey duration is only 7 minutes, which is too short to a considerate answer. Even worse, the distribution of survey duration is extremely right-skewed with a maximum of 34586 and a mean of 1049. We suspect some respondents went to 
do other things while remaining signing-in the survey page. The unreasonably long survey duration does not convince us that the answers were carefully produced. To address this attentiveness concern, we define a Reasonable duration sample, in which we only keep the respondents whose documented survey duration is between 15-60 minutes. Finally, we use the intersection between Narrow time window sample and Reasonable duration sample and the intersection between Proximity sample and Reasonable duration sample to make two more subsamples. ${ }^{4}$ Plus Full sample, we have 6 samples in total prepared for analysis.

Table 1 shows the percentage of gun control legislation supporters before and after the Orlando shooting. In all samples, the percentage of gun control legislation supporters increased following the shooting. In the next section, for each of our 6 samples, we run two logit models (bivariate and full specification) for estimation. We apply the Internet-sample adjusted weight, which is provided by the survey team in the original dataset, to make our results as representative to the population (English-speaking adults in the US) as possible. Besides logit models, we also run linear probability models to ensure that any results we have are not model-dependant.

Table 1: Percentage of gun control legislation supporters before and after the 2016 Orlando shooting

\begin{tabular}{lcc}
\hline & Pre-shooting & Post-shooting \\
\hline Full sample & 53.789 & 62.017 \\
Narrow time window & 55.914 & 64.000 \\
Proximity & 54.651 & 61.538 \\
Reasonable duration & 52.568 & 59.580 \\
Narrow time window $\cap$ Reasonable duration & 52.239 & 58.696 \\
Proximity $\cap$ Reasonable duration & 54.412 & 56.410 \\
\hline
\end{tabular}

Note: See Appendix Table 5 for their sample sizes.

\section{Results}

We find the 2016 Orlando shooting did not elicit statistically significant attitudinal change in favor of gun control legislation among Americans. Given the binary nature of our treatment variable, we use the differenced predicted probabilities to present our substantive effects. When doing so

4. We do not use the intersection between Narrow time window sample and Proximity sample because doing so leaves too small sample size. 
based on our full specification, we hold the control variables fixed at their median values. Figure 1 shows our bivariate specification results while Figure 2 shows our full specification results. When the Full sample is used, the differenced predicted probabilities are positive, meaning that the probability of supporting gun control legislation is higher among Americans after the Orlando shooting. However, even the $90 \%$ confidence interval goes across the value of 0 , suggesting this gun control legislation support difference is not statistically significant. When we use our subsamples, the confidence intervals still go across 0 . It means even we only focus on the respondents who were most likely to remain in the treated status (Narrow time window sample), who lived close to and thus likely to pay attention to the shooting (Proximity sample), and who showed more attentiveness during the survey (Reasonable duration sample), and their intersections, we still find no sufficient statistical evidence to claim that Americans are responsive to mass shooting.

In binary response models, unbiased coefficients not necessarily translate into unbiased quantities of interest (Rainey 2017). So, only relying on the confidence intervals of our differenced probabilities for statistical inference might be problematic. We therefore also report conventional regression tables underpinning Figure 1 and Figure 2 in Appendix Table 6-11. The statistical insignificance of our treatment indicator (Post-shooting) confirms our null finding is not a product of transformation-induced bias as discussed in Rainey (2017). When we use linear probability models instead of logit models, our results are substantively the same (see Appendix Table 12-17). It suggests our null finding is not dependent on any single statistical model or its assumptions.

We finally use the exact same statistical routine as RT did-running the bivariate specification based on subsamples defined by respondents' personal characteristics. We use our control variables to define these subsamples. ${ }^{5}$ We do not follow this approach for our main results since under the covaraite imbalance problem we have, simply doing so would lead us to suffer from non-random sample selection issue. However, we present this approach's results here to show that our null finding is not an outcome of any single way of statistical analysis. Also, since we aim to extend RT's study, presenting the results based on their approach is also helpful for cross-study comparison. Appendix Table 17 shows these results. Our treatment indicator is not statistically significant even at the $\alpha=0.10$ level among all of the 12 subsamples defined by our 6 control

5. These subsamples are produced on the basis of our Full sample. 
Figure 1: Differenced predicted probabilities of gun control legislation support (Pre-shooting versus Post-shooting), bivariate specification

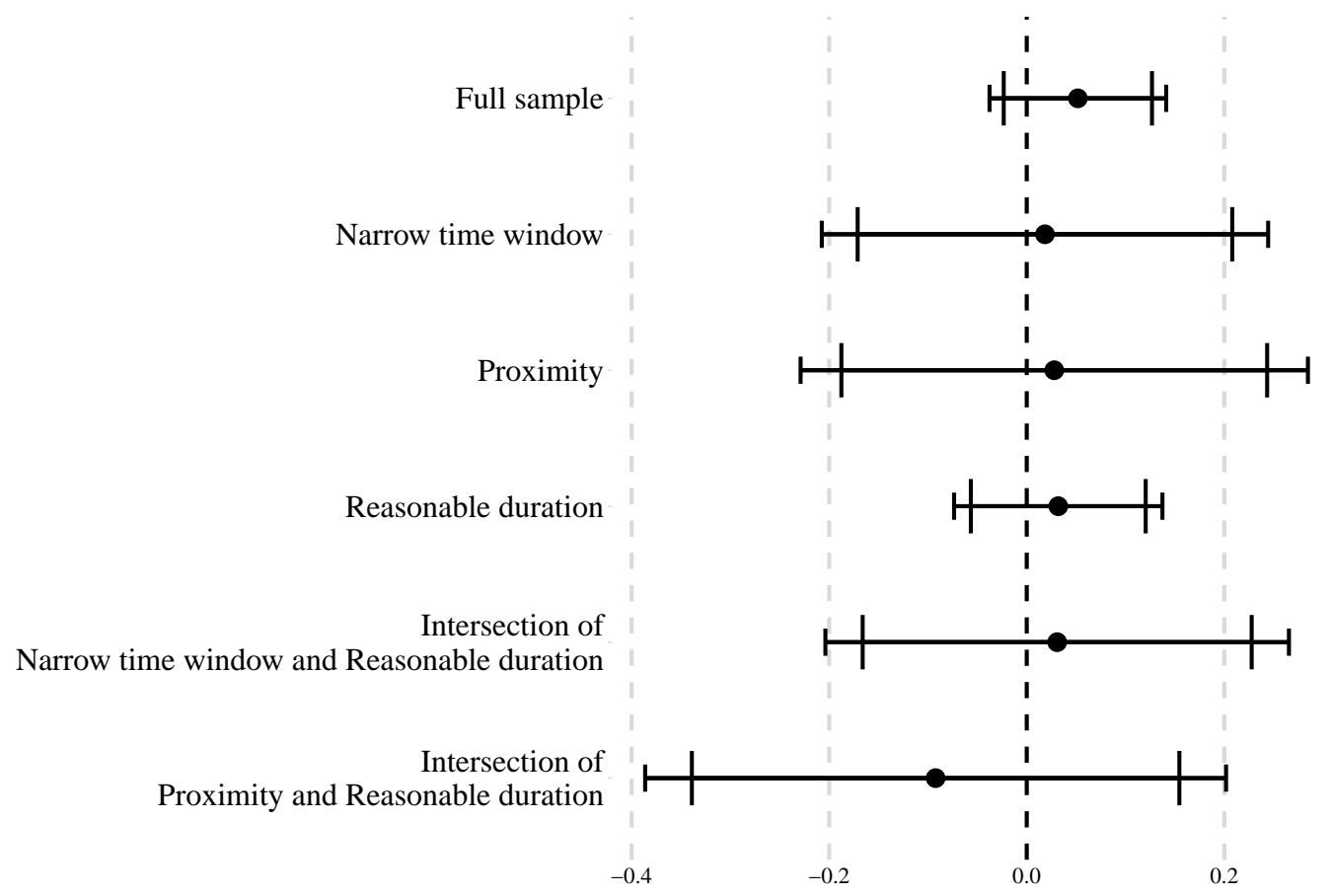

Note: Wider error bars indicate the $95 \%$ confidence intervals while narrower error bars indicate the $90 \%$ confidence intervals. The standard errors necessary for calculating the confidence intervals are derived from the Delta method.

variables. ${ }^{6}$ This means the 2016 Orlando shooting were unable to influence American's enough attitudinal change in favor of gun control, even among the people whose predisposition are more aligned to progressive gun policy, such as parents and liberals.

\section{Conclusion}

The repeatedly happening mass shootings and the deadlocked gun control issue in the US puzzles scholars while taking too many lives away. In this research note, we ask are mass shootings able to galvanize public support for gun control? We use TAPS wave 55 data and employ the unexpected-event-during-survey design to answer this question. By comparing the respondents who completed the survey before and after the occurrence of the 2016 Orlando shooting, we find such a tragic gun violence event did not elicit American's statistically distinguishable attitudinal change in favor of gun control legislation. This null finding holds when we only use the respon-

6. For Political interest, we code very as 1 while all others as 0 . For Ideology, we code 1-3 as liberal and 4-6 as conservative. So that now our 6 control variables are all binary and we have 12 subsamples defined by them. 
Figure 2: Differenced predicted probabilities of gun control legislation support (Pre-shooting versus Post-shooting), full specification

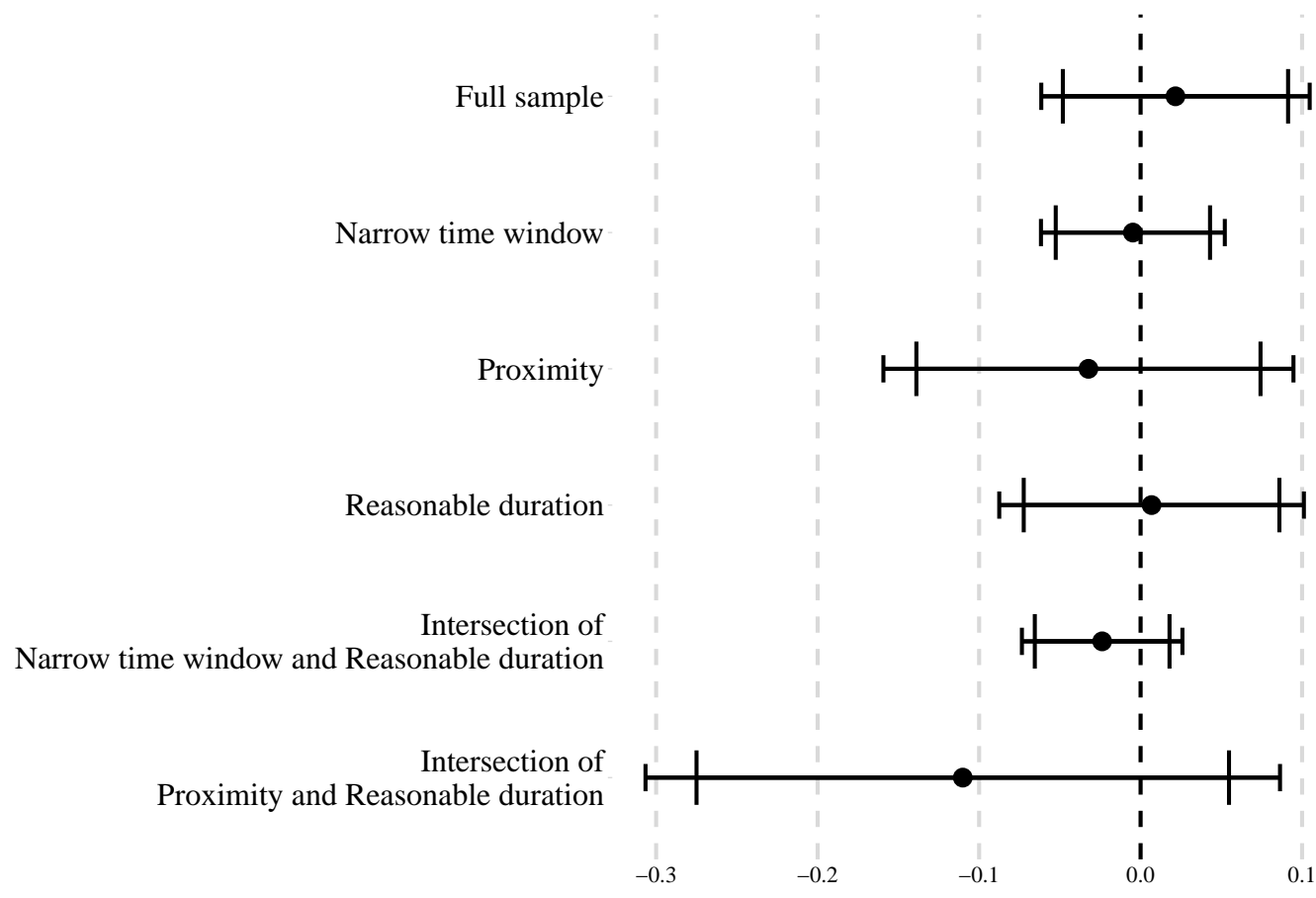

Note: Covariates are held at their median values. Wider error bars indicate the $95 \%$ confidence intervals while narrower error bars indicate the $90 \%$ confidence intervals. The standard errors necessary for calculating the confidence intervals are derived from the Delta method. 
dents who (a) participated the survey barely one day before and after the shooting, (b) lived near Orlando, (c) completed the survey within a reasonable duration, and (a) $\cap$ (c) and (b) $\cap$ (c). Our results contribute to the literature by adding new evidence demonstrating mass shootings fail to influence American's attitudinal change towards gun control support (Rogowski and Tucker 2019; Barney and Schaffner 2019). This counterintuitive finding partly explains why there is no significant progress in gun control policy change in the US, even the repeatedly happening mass

shootings have been causing too many tragedies. Future research may consider to explore the mechanism underlying American's nonresponse to mass shootings.

\section{References}

Barney, David J, and Brian F Schaffner. 2019. "Reexamining the Effect of Mass Shootings on Public Support for Gun Control." British Journal of Political Science 49 (4): 1555-65.

Hartman, Todd K, and Benjamin J Newman. 2019. “Accounting for Pre-Treatment Exposure in Panel Data: Re-Estimating the Effect of Mass Public Shootings." British Journal of Political Science 49 (4): 1567-76.

Muñoz, Jordi, Albert Falcó-Gimeno, and Enrique Hernández. 2020. “Unexpected Event during Survey Design: Promise and Pitfalls for Causal Inference." Political Analysis 28 (2): 186-206.

Newman, Benjamin J., and Todd K. Hartman. 2019. "Mass Shootings and Public Support for Gun Control." British Journal of Political Science 49 (4): 1527-53.

Rainey, Carlisle. 2017. "Transformation-induced Bias: Unbiased Coefficients Do Not Imply Unbiased Quantities of Interest." Political Analysis 25 (3): 402-9.

Rogowski, Jon C., and Patrick D. Tucker. 2019. "Critical Events and Attitude Change: Support for Gun Control After Mass Shootings." Political Science Research and Methods 7 (4): 903-11. 